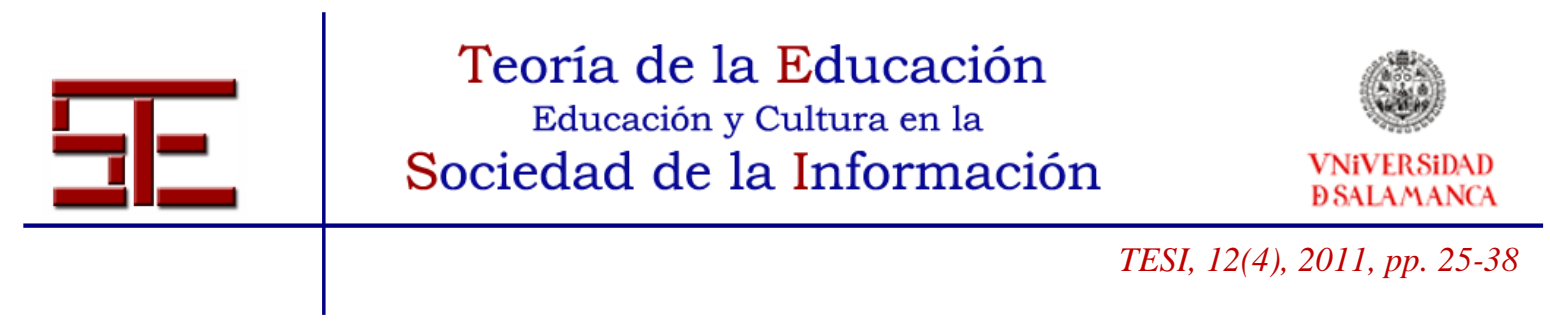

\title{
NIÑOS 2.0, UNA EXPERIENCIA FORMATIVA EN ACTITUDES Y VALORES PARA EL PROFESORADO ANTE LA WEB 2.0 Y TIC
}

Resumen: Niños 2.0 es una experiencia formativa para el profesorado que trabaja con niños, basada en actitudes y valores que fomentan el uso de las herramientas de la Web 2.0 y las TIC. Las actitudes aplicadas tienen que ver con superar los miedos, emprender la cultura 2.0 y ser un profesor presumer; conocer, incorporarse e interaccionar en la Web 2.0; desaprender, actualizarse y cambiar a las nuevas metodologías de aprendizaje colaborativo con la tecnología del aprendizaje y del conocimiento; proteger y supervisar como educadores el acceso de menores a la Red; crear y aportar ejercitándose en Talleres 2.0, centrados en servicios y aplicaciones Web, redes sociales, videoclips educativos, Wikis, blogs, mashups, folcsonomía y pizarra digital. Esta acción formativa se desarrolló en unas Jornadas realizadas en Magisterio de la UPSA en abril de 2011, contando con la participación de expertos y con la buena valoración de los participantes.

Palabras clave: Niños 2.0; Web 2.0; TIC; actitudes; formación profesorado; PLE.

Fernando González Alonso, Manuel Carretero González. Iacinto Escudero Vidal v Olga Arranz García 


\title{
CHILDREN 2.0, A FORMATIVE EXPERIENCE IN ATTITUDES AND VALUES FOR TEACHERS TO WEB 2.0 AND TIC
}

\begin{abstract}
Children 2.0 is a formative experience for teachers working with children, based on attitudes and values that encourage the use of the tools of Web 2.0 and ICT. Attitudes which worked relate to overcome fears, undertake the culture 2.0 and becoming a teacher presumer; know, incorporate, and interact on the Web 2.0; unlearn, updated, and switch to new methodologies of collaborative learning with learning and knowledge technology; protect and monitor as educators access by minors to the network; create and contribute by exercising in workshop 2.0, focusing on services and Web applications, social networks, educational video, Wikis, blogs, mashups, folksonomy, and whiteboard. This formative action took place in a Conference in April 2011, in the UPSA, with the participation of experts, and with the good assessment of the participants.
\end{abstract}

Keywords: Children 2.0; Web 2.0; ICT; attitudes; teacher training; PLE

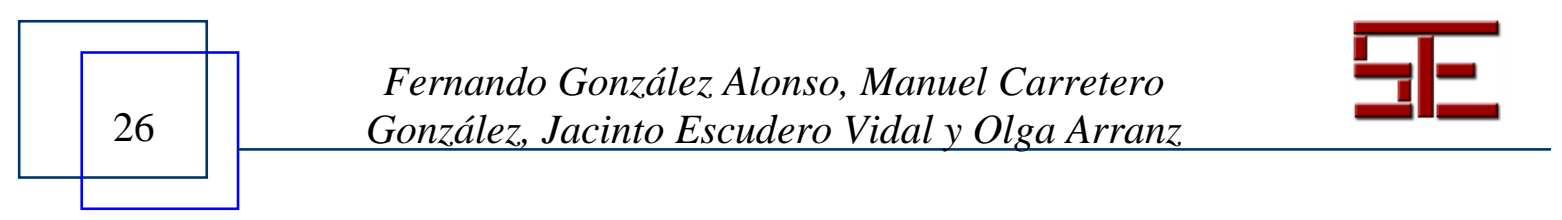




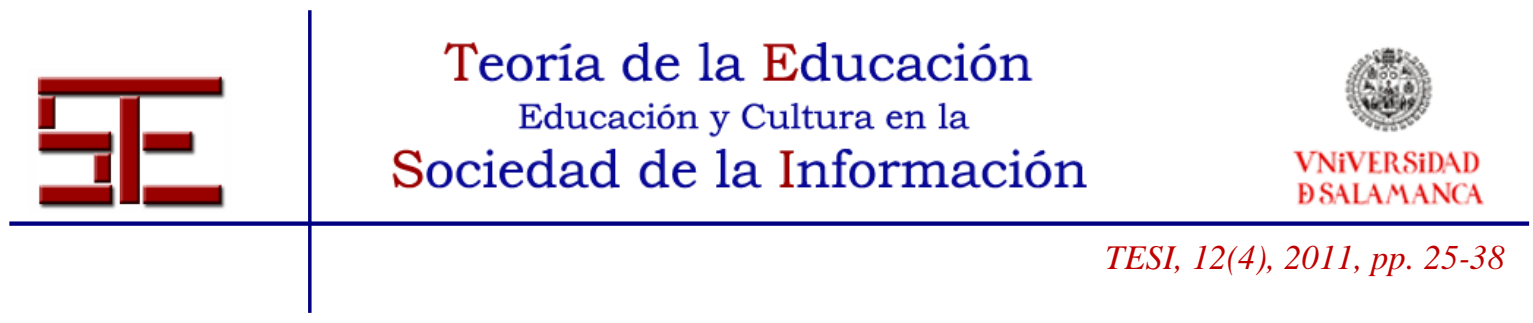

\section{NIÑOS 2.0, UNA EXPERIENCIA FORMATIVA EN ACTITUDES Y VALORES PARA EL PROFESORADO ANTE LA WEB 2.0 Y TIC}

Fecha de recepción: 27/10/2011; fecha de aceptación: 11/12/2011; fecha de publicación: 20/12/2011

Fernando González Alonso

fgonzalezal@upsa.es

Universidad Pontificia de Salamanca

Manuel Carretero González

mcarreterogo@upsa.es

Universidad Pontificia de Salamanca

Jacinto Escudero Vidal

jescuderovi@upsa.es

Universidad Pontificia de Salamanca

Olga Arranz García

oarranzga@upsa.es

Universidad Pontificia de Salamanca

\section{1.- INTRODUCCIÓN}

En el escrito "Propuestas para la renovación de las metodologías educativas en la Universidad", elaborado bajo petición del MEC, se diseña el camino para cimentar el Espacio Europeo de Educación Superior (EEES) en el que estamos inmersos como:

La oportunidad perfecta para impulsar una reforma que no debe quedarse en una mera reconversión de la escritura y contenidos de los estudios, sino que debe alcanzar al meollo de la actividad universitaria, que radica en la interacción profesoresestudiantes para la generación del aprendizaje. (Consejo de Coordinación Universitaria, 2006, 7).

Esta renovación metodológica universitaria con el EEES de fondo se hace especialmente importante con el uso y apoyo de la Web 2.0 y las TIC, máxime cuando se ha de formar y actualizar al profesorado en recursos que su alumnado intuye. En el caso de los maestros cobra especial sentido, pues sus niños y niñas 2.0 son los que tienen en sus aulas y grupos. Son los que padres y madres tienen en sus hogares. Y lo

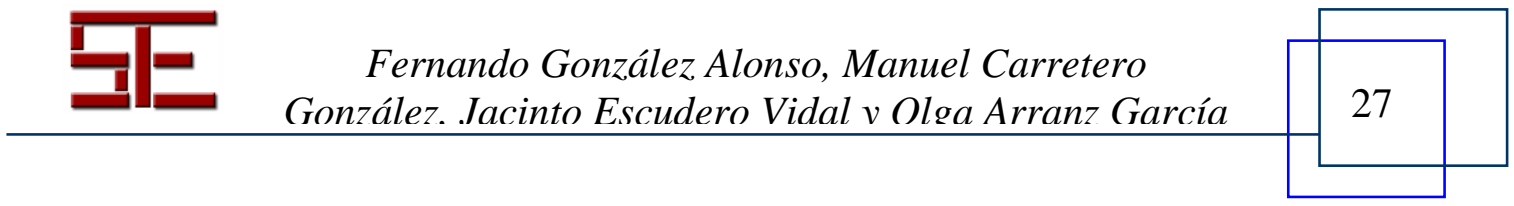




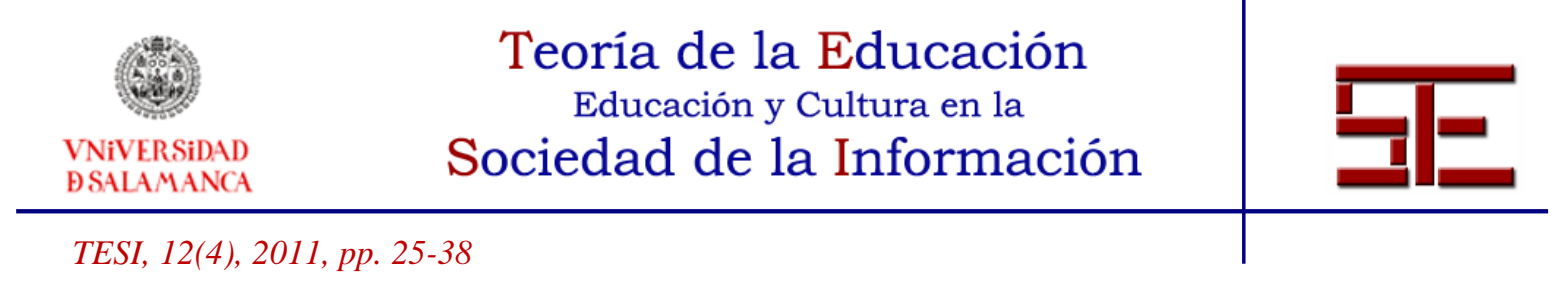

son porque han nacido con la red y muestran intuición e interés en la competencia digital. Son la generación 2.0. Niños y jóvenes se convierten en protagonistas del cambio educativo, con la aparición de recursos informáticos y digitales que revolucionan la comunicación. Internet se ha convertido en prototipo y medio necesario para todos los ámbitos de la vida, donde la educación, el aprendizaje, la información y la comunicación destacan por propio peso. Desde muy pequeños, Internet es para los niños y niñas una herramienta intuitiva, entretenedora, informativa y lúdica, para estimular su aprendizaje.

El reto y responsabilidad de padres y educadores es aprender y enseñar a vivir y convivir en la Red. Los maestros y profesores, y la escuela en general, guardan cierto temor e inseguridad ante ese nuevo mundo, y una respetable distancia ante esta realidad que en ocasiones incomoda. Era necesario asumir algunas actitudes que superen el escepticismo y las resistencias al cambio, y fomenten la observación, el interés, la participación y la colaboración (Gisbert, 2004).

Estas actitudes y valores han estado de fondo en las Jornadas denominadas "Niños 2.0" y con el subtítulo "emprender la actitud y cultura 2.0", realizadas en abril de 2011 en Salamanca, organizadas por el Instituto de Estudios Maristas (IEM) y Magisterio de la Facultad de Ciencias Humanas y Sociales de la Universidad Pontificia de Salamanca.

\section{2.- OBJETIVOS}

Objetivos de esta acción formativa fueron constatar el análisis de esta realidad; el necesario espíritu emprendedor ante la cultura 2.0; el enfrentar los miedos y adaptarse a los cambios; el conocer y acceder a la Web 2.0 y sus herramientas, entre ellas las redes sociales; el aprender cómo proteger a los menores en su acceso a la Red; con la necesaria supervisión y uso de adecuados filtros; constatar, en definitiva, la distancia que los educadores y la escuela muestran ante los niños; actualizarnos y optar por los necesarios cambios metodológicos para aprender colaborativamente a través de la Web 2.0 y las TIC en los diferentes ámbitos educativos.

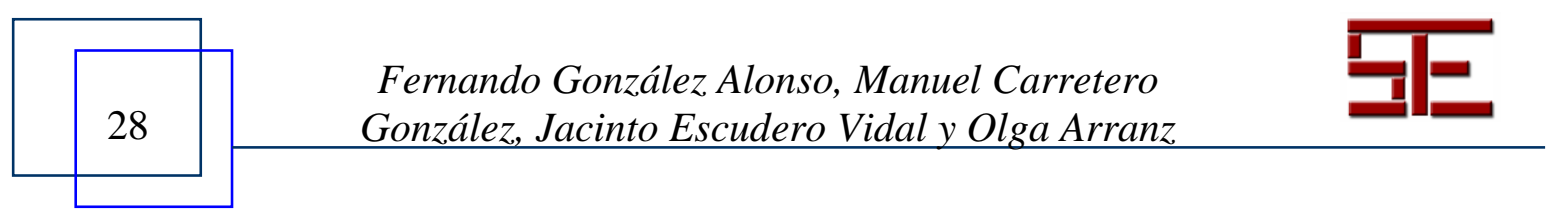




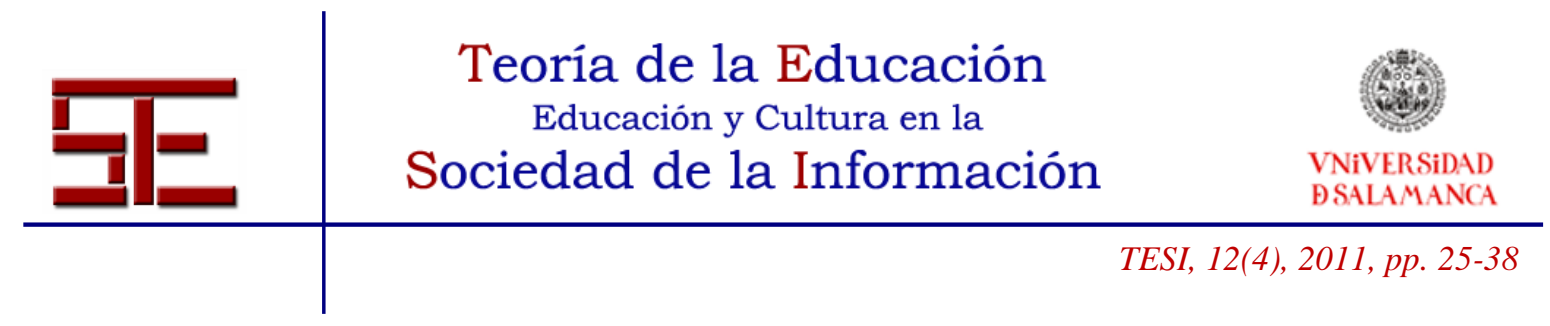

\section{3.- ACTITUDES PARA EL PROFESORADO ANTE LA WEB 2.0 Y LAS TIC}

Las actitudes forman parte de los programas de integración de las nuevas tecnologías de la información para el aula, ya que los profesores hacen de ellas una experiencia de enseñanza para la integración en la misma (Ajzen; Fishbein, 1977).

Debido al desarrollo tecnológico alcanzado en la sociedad actual, la utilización de la tecnología en general y de los ordenadores, en particular, estimula que la mayor parte de las personas desarrollen con ellos algún tipo de actitud y de valor (Sánchez García, 2011).

Entendemos por actitudes la forma que tiene una persona de actuar, de comportarse, lo que muestra y lo que la motiva para enfrentarse a las cosas. La orientación en la formación del profesorado ante los cambios de aprendizaje era necesaria para plantear algunas actitudes concretas para reflexionarlas y aplicarlas desde las ponencias y talleres de las Jornadas, como se puede ver en la Figura I: analizar la realidad y enfrentarse a ella sin miedos; emprender la cultura 2.0 y ser presumer; conocer, incorporarse e interaccionar; proteger y supervisar como educadores, el acceso de menores a la red; desaprender, actualizarse y cambiar a nuevas metodologías de aprendizaje colaborativo; y crear y aportar ejercitándose en Talleres 2.0.

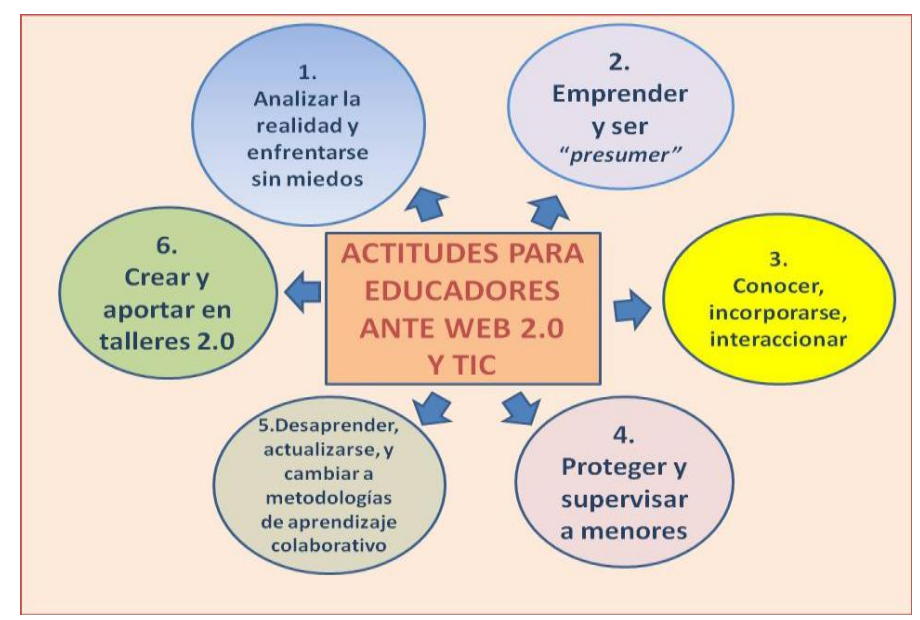

Figura 1: Actitudes para educadores ante el acceso a la Web 2.0 y TIC.

Elaboración propia.

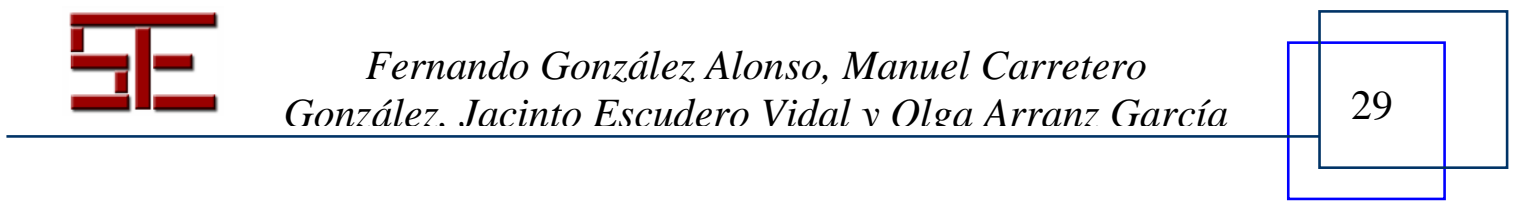




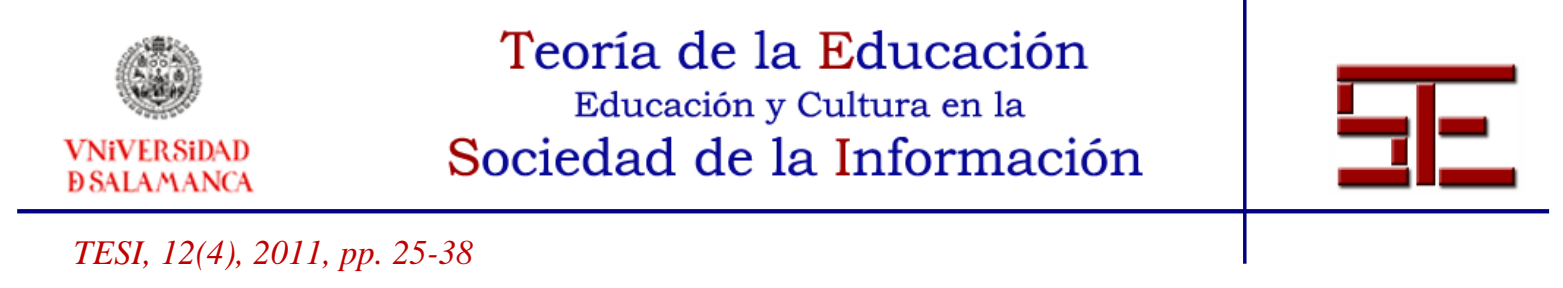

\section{1.- Actitud 1: Analizar la realidad y enfrentarse a ella sin miedos}

La primera actitud planteada es la de analizar la realidad personal y profesional del profesor: mirarla, observarla y enfrentarse a ella sin miedos. Sólo se puede vivir desde el amor o desde el miedo. Vivir sin miedos es deseable y posible. Tenemos que hacer como el águila, un profundo proceso de transformación y de cambio. Necesitamos una nueva manera de mirar la realidad, un nuevo paradigma, donde desaprendamos costumbres, ideas y temores (Fernández, 2010).

Se hace necesario para el profesorado afrontar el cambio de la nueva época con energía renovada, haciéndonos las preguntas claves, cuyas respuestas tengan el poder transformador para vivir desde la honestidad, la valentía y la consciencia, sintonizando con los cambios más significativos tecnológicos y de la Web 2.0, para que el profesorado se sienta más feliz y comprometido con la nueva realidad.

Éste es el planteamiento que en la ponencia Vivir sin miedos para adaptarse a un mundo cambiante se realizó ${ }^{1}$ para abrir la reflexión de la realidad personal y del afrontamiento de miedos y resistencias al cambio.

\section{2.- Actitud 2: Emprender la cultura 2.0 y ser presumer}

El proceso de alfabetización digital, incorporación de los nuevos lenguajes, participación en Internet suponen un recorrido por parte de los docentes, que no siempre resulta fácil. La cultura y actitud 2.0 supone no sólo unas ciertas destrezas tecnológicas, sino un verdadero cambio.

Ser docente para los niños 2.0 significa pasar de la pasividad en el consumo de la información en Internet a la proactividad en la construcción de sus contenidos. El docente actual, tanto si es nativo digital o inmigrante, necesita dar el salto que le convierta en protagonista de la red, ayudando a clasificar, juzgar e incorporar la información. Ha de ser responsable de dinamizar la colaboración entre usuarios, esforzándose por estar a la vanguardia de los usos emergentes que nacen de una nueva cultura en la Web, la cultura del educador prosumer, término que viene del acrónimo de producer y consume, que es quien interactúa en la educación con herramientas Web 2.0, en la producción de información que circula principalmente en blogs y podcast.

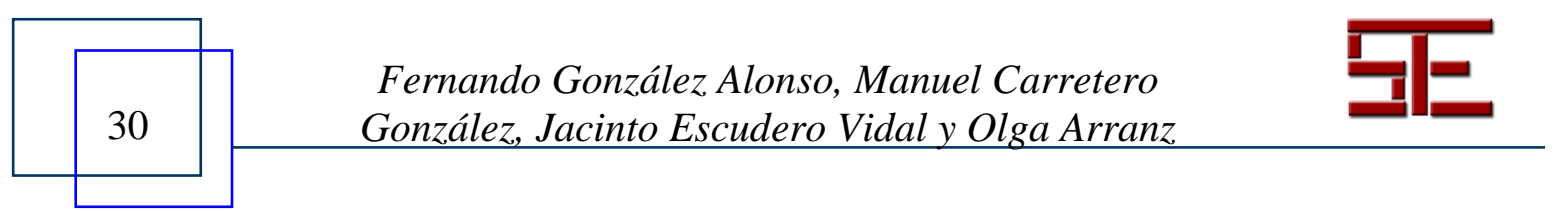




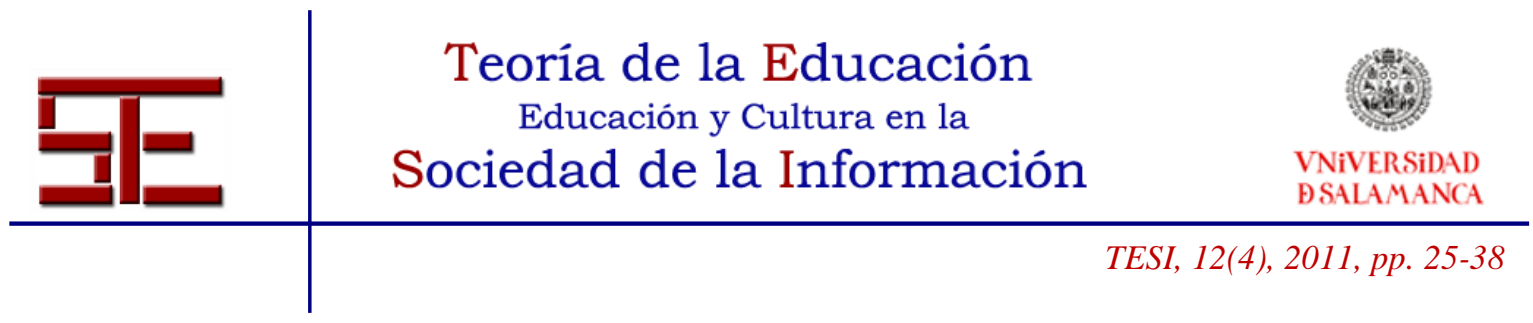

Otra reflexión se llevó a cabo ${ }^{2}$ con el título Emprender la cultura 2.0, que es la actitud del educador presumer, que construye, participa y está conectado, sin condicionantes físicos arquitectónicos o de mobiliario. Es la actitud ante la interacción, como espacio donde se producen la comunicación y el aprendizaje 2.0 con adecuadas estrategias educativas. Esta actitud emprendedora y presumer, exige esfuerzo, reaprendizaje y alfabetización visual y digital, como veremos más adelante.

\section{3.- Actitud 3: Conocer, incorporarse e interactuar en la Web 2.0}

Varios estudios elaborados en el contexto de la universidad española como el de Alba (2004), Barros (2004), Michavila (2004), o el Consejo de Coordinación Universitaria ya citado (2006) indican que las TIC son agentes importantes para el armazón del cambio metodológico (Del Moral; Rodríguez, 2008).

A partir de 2004 la expresión Web 2.0 se introdujo en el ámbito de las TIC a partir de una conferencia organizada por la editorial $O^{\prime}$ Reilly Media, término normalizado después por Dale Dougherry, inventor del término Web 2.0 (De Pablos, 2008). Dicha Web 2.0 se refiere a conceptos, tecnologías y actitudes hacia esas tecnologías y sus aplicaciones con herramientas 2.0 asociadas a Google, GMail, Blogger, Wikipedia, Flickr o Firefos, apoyadas en metalenguajes y estándares de representación, que mejoran la gestión de la información, la usabilidad, la interacción y el cambio de mentalidad entre los usuarios (Anderson, 2007).

En este sentido hay que formar en la Universidad al profesorado en la Web 2.0 para "crear, diseñar, elaborar, colaborar, publicar en línea, facilitar un aprendizaje más autónomo, participar en actividades grupales, aumentar el interés y la motivación del alumnado, desarrollar y mejorar las competencias digitales, y realizar nuevas actividades del aprendizaje" (Fernández Sánchez, 2011, 788). Esta formación es fundamental en la educación superior como formación inicial, continua y permanente (Baelo; Arias, 2011).

Por estas razones se explicó en otra ponencia ${ }^{3}$ lo que son la "Web 2.0 y las redes sociales", para comprender mejor las posibilidades que encierran, poder aplicarlas en el ámbito educativo para beneficio de un alumnado más motivado y participativo, y, a nivel social, reconocer la importancia que tiene, por ejemplo, en momentos como en el hundimiento del petrolero Prestige, o en acontecimientos recientes como los acaecidos

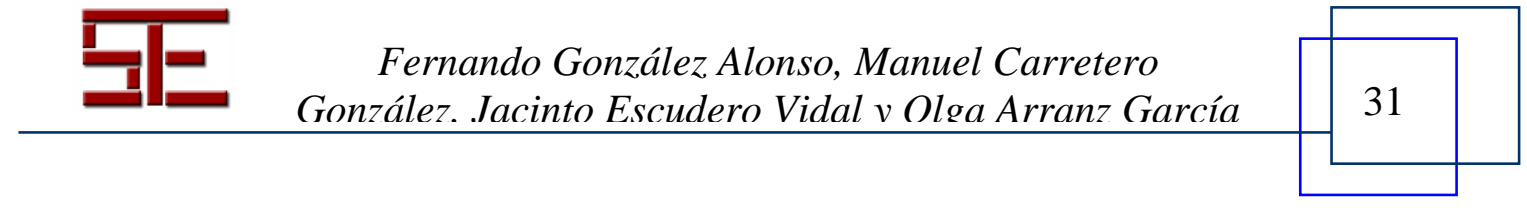




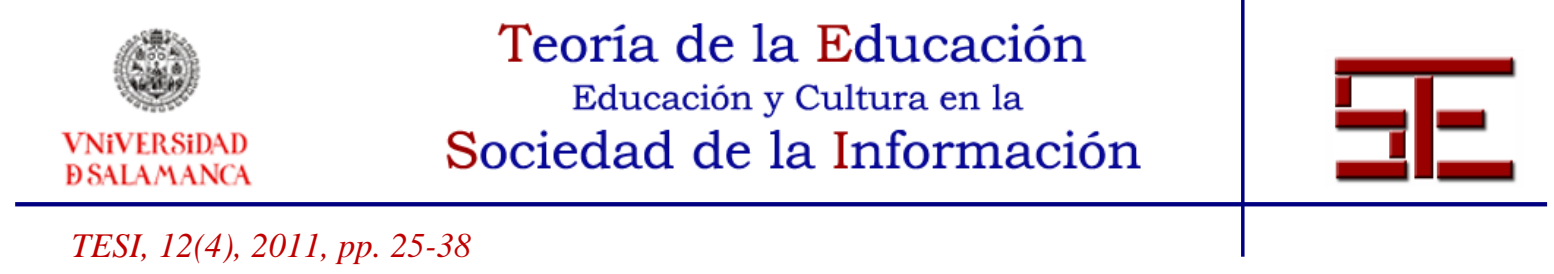

en las revueltas árabes, o las concentraciones del movimiento del $15-M$ en España y otros países

\section{4.- Actitud 4: Proteger y supervisar el acceso de los menores a la Red}

Objetivo de esta actitud era dar a conocer la formación necesaria que han de tener padres y educadores en general, para enseñar sobre todo a los menores, el uso de Internet, donde viven mucho tiempo; en la aplicación de normas y filtros, y en el conocimiento de leyes, estrategias y procedimientos para la navegación segura, para el conocimiento de nuevos delitos en la red como acoso, amenazas, chantaje, suplantación de identidad, phising, ciberbuylling; la protección del ordenador y Webcams, el uso seguro de correo electrónico, redes sociales, teléfonos móviles, etc.

Todos estos elementos se abordaron de forma extensa ${ }^{4}$ en la conferencia Hacker para educadores: Protección de menores en la red.

3.5.- Actitud 5: Desaprender, actualizarse y cambiar a nuevas metodologías de aprendizaje colaborativo y PLE

Desaprender respecto de recursos y métodos tradicionales: papel, charla, lección. Actualizarse y reaprender como inversión a través de foros, perfiles, twitt, comentarios, y cambiar a la participación, intervención y uso de adecuada identidad digital.

Con el uso de las TIC y la incorporación de las TAC, Tecnologías del Aprendizaje y de la Comunicación, nos permiten gestionar el conocimiento a través de herramientas 2.0 y la incorporación de las metodologías de aprendizaje colaborativo.

El proceso que nos lleva a identificar las herramientas que utilizamos, las personas con las que nos relacionamos, los lugares en los que lo hacemos, los contenidos que generamos o la información que transmitimos constituye nuestro entorno personal de aprendizaje: PLE (Personal Learning Environment).

El PLE es un sistema que ayuda a profesores y alumnos a controlar y gestionar su propio aprendizaje, fijando sus propios objetivos, gestionando los contenidos y procesos, y comunicando con otros su propio aprendizaje.

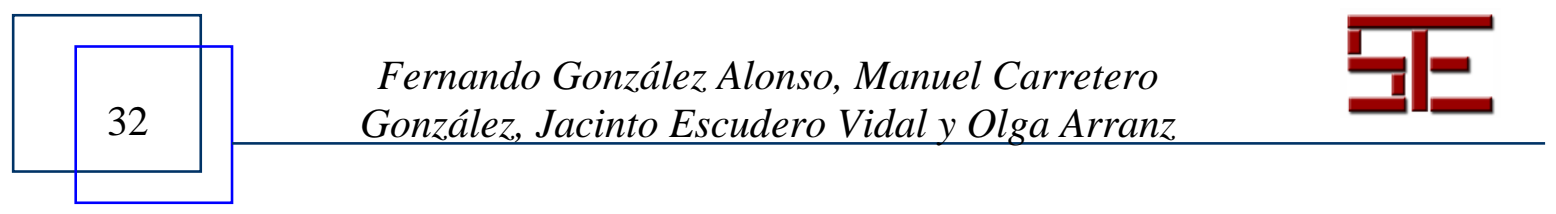




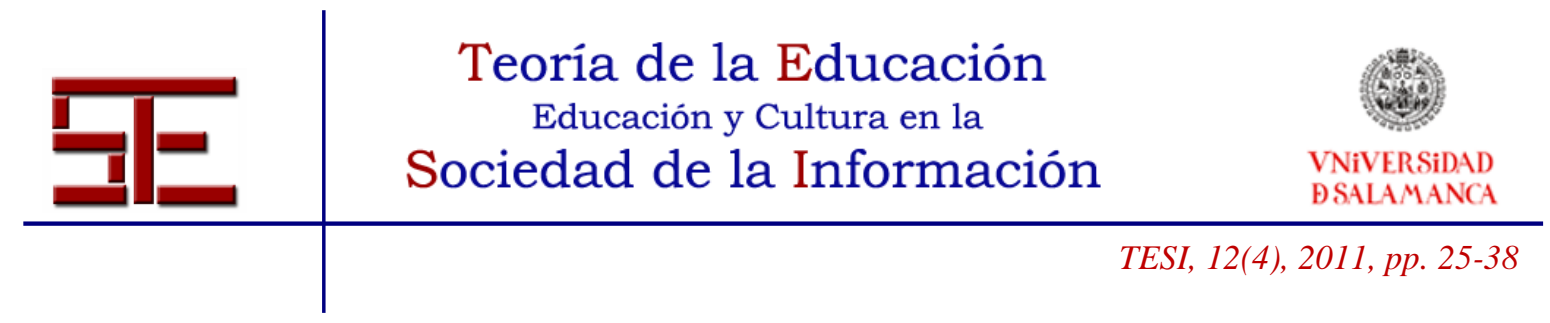

A través de una aplicación de escritorio, un PLE puede estar compuesto de uno o varios subsistemas que pueden integrar el uso de redes sociales, servicios web, sindicación de contenidos, para conectar una serie de recursos y sistemas, dentro de un espacio gestionado personalmente.

El PLE de hace décadas estaría constituido por el aula, cuaderno, lápices, profesor, etc., en el PLE actual del profesor, Internet y sus recursos educativos 2.0 son los protagonistas.

La ponencia TIC - TAC: Formas de enseñar versus maneras de aprender, mostró ${ }^{5}$ estos importantes cambios que se están produciendo entre las formas de enseñar y las nuevas maneras de aprender colaborativamente con el apoyo de las herramientas 2.0, conformando nuestro actual PLE.

\section{6.- Actitud 6: Crear y aportar ejercitándose en Talleres 2.0}

Con espíritu deportivo es necesario entrenarse y ejercitarse con actitud creativa aportando en talleres lo que se ha ido aprendiendo y asumiendo en las sesiones formativas anteriores.

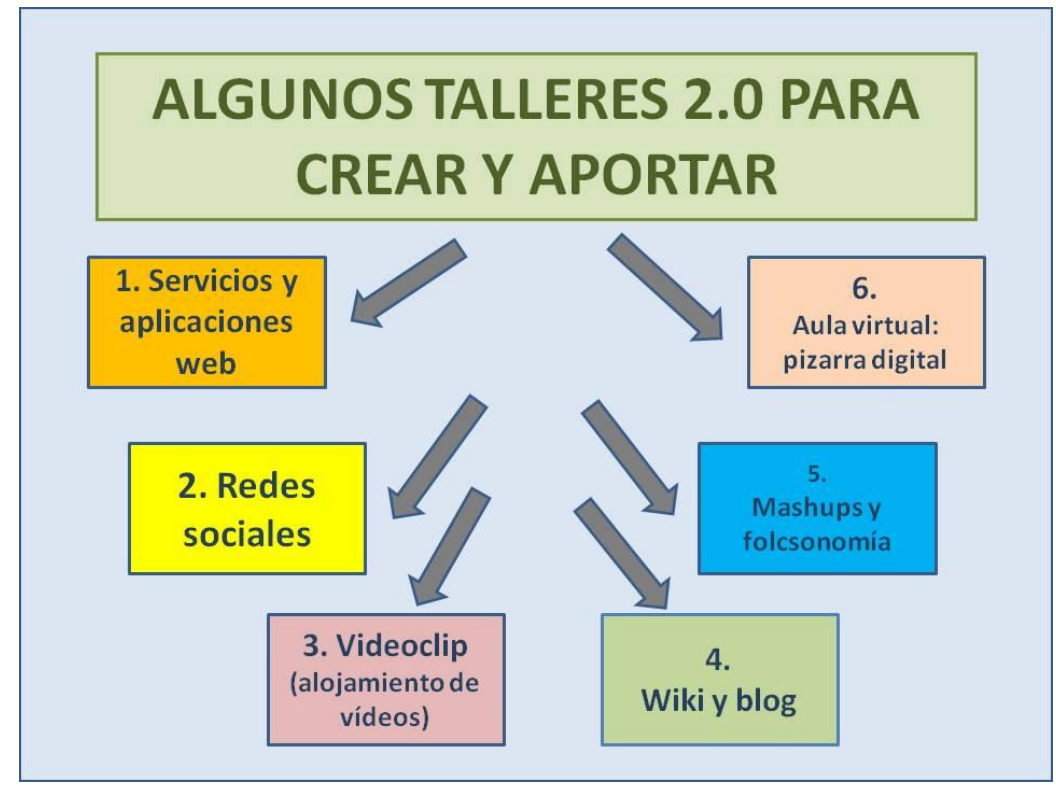

Figura 2: Talleres 2.0 para crear y aportar en la formación del profesorado. Elaboración propia.

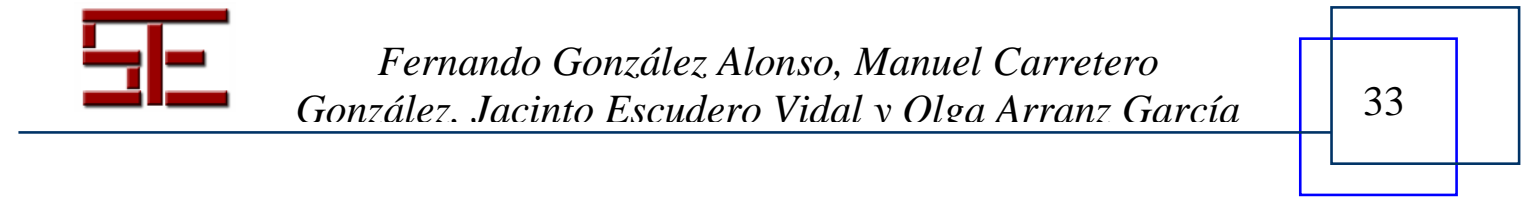




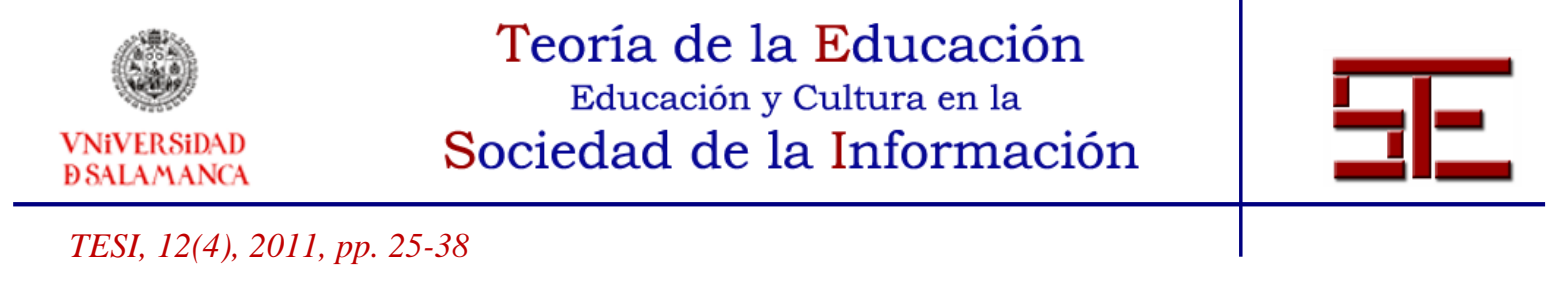

Los Talleres 2.0 fueron dirigidos por otros tantos expertos, y versaron sobre servicios y aplicaciones web, redes sociales, videoclips educativos, Wikis, blogs, mashups, folcsonomía y pizarra digital.

Taller 1: "Servicios y aplicaciones Web"

El taller se basó en el conocimiento de la Web 2.0 aplicada a la educación, las mejores herramientas 2.0 y la realización de casos prácticos con herramientas seleccionadas tales como facebook, twitter, flickr, youtube, slideshare, blogs, wiki, delicious, scribd, linkedin, xing.

\section{Taller 2: "Redes Sociales"}

En él se analizó la gestión de la privacidad en las Redes, por qué y para qué se utilizan, las condiciones de uso, la gestión de la identidad digital, ejemplos de aplicaciones educativas de Redes Sociales y sugerencias de lugares interesantes en los que estar como educadores.

\section{Taller 3: "Video clip exprés"}

Con pocos recursos y mucha creatividad, los participantes se convirtieron en insólitos artistas digitales. Los pasos para la realización del videoclip empezaron con una lluvia de ideas y presentación de recursos, elección del tema, grabación, secuenciación, importación, edición, y a visualizar.

\section{Taller 4: "Wikis y blog"}

El desarrollo de las tecnologías ha facilitado el acceso a grandes cantidades de información, al principio por personas e instituciones privilegiadas que accedían a la red, y actualmente por todos los que utilizan aplicaciones como la wiki y el blog, que han universalizado la creación y publicación en la red, dándose a conocer en este taller. La particularidad de una wiki es la colaboración entre varias personas para generar un único documento, en el que todos pueden aportar. Por su parte los blogs, como publicaciones on line que son, se actualizan con alta periodicidad en orden cronológico inverso por un único autor. Los lectores pueden hacer comentarios manteniendo un diálogo con el escritor, pero sin modificar lo publicado. Hay blogs educativos y Wikis muy variadas que se dieron a conocer.

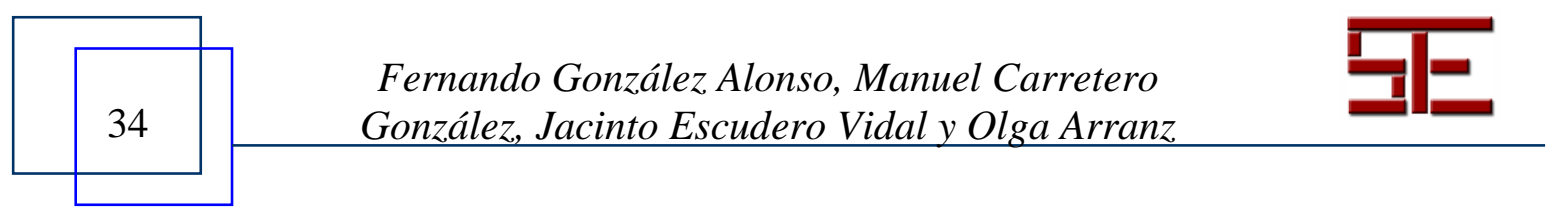




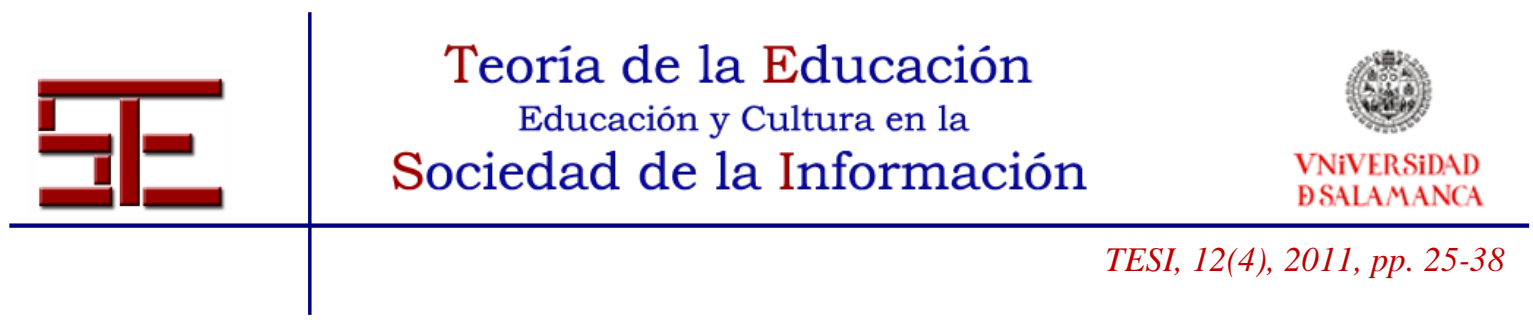

\section{Taller 5: "Aula virtual: pizarra digital"}

En este taller se pretendió conocer el uso técnico y metodológico de la pizarra digital interactiva (PDI), a través de los pasos: aulas interactivas, pizarra digital interactiva (Smart Board), y sistema de presentaciones notebook.

Un estudio reciente, entre otros factores, concluye sobre el uso de las TIC en el aula, como las pizarras digitales, que en los profesores aumentan la motivación para enseñar y en el alumno para aprender, aunque en muchos casos se restringe al juego educativo, o como fuente de información (Sánchez; Pinto; González, 2011).

\section{Taller 6: "Mashups y folcsonomía"}

En el taller se dio a conocer que los mashups son sitios que beben de recursos externos que se pueden enlazar de diferentes maneras, aportando al contenido del autor, una amplia variedad de posibilidades. Para llegar a estos recursos, es fundamental una buena organización y clasificación de los mismos. Los usuarios, sólo por el hecho de navegar por determinados sitios, favorecemos que determinadas webs se ubiquen en mejor posición en los buscadores. Además, muchos sitios nos permiten agregar etiquetas o realizar votaciones sobre sus productos. Poder participar de la folcsonomía y aprender de ella, ayudó a los participantes a llegar a contenidos de mejor calidad y a que otros también puedan hacerlo.

\section{4.- CONCLUSIONES}

Se hace necesario fomentar y favorecer la formación de educadores en general, basada en actitudes que impulsen y familiaricen el uso de las TIC y Web 2.0. Se constata la distancia entre niños y niñas que están en su hábitat tecnológico natural, en el que han nacido y se mueven con intuición, con los maestros, profesores y padres que han de hacer el esfuerzo por desaprender y reaprender competencias digitales educativas.

Se confirma la necesidad de las instituciones educativas por organizar acciones formativas como la experiencia de las Jornadas Niños 2.0 que se presenta, basadas en ponencias y experiencias prácticas de talleres de formación. Los métodos y la planificación formativa de docentes es prioritaria sobre la existencia de la tecnología educativa como tal. Se ve necesario crear espacios y tiempos que favorezcan el crecimiento creativo, para aportar experiencias y producir nuevos aprendizajes con metodologías colaborativas en la educación superior con los futuros formadores.

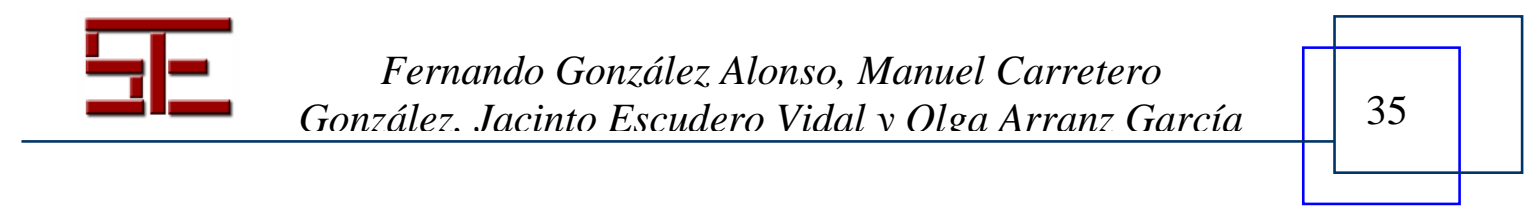




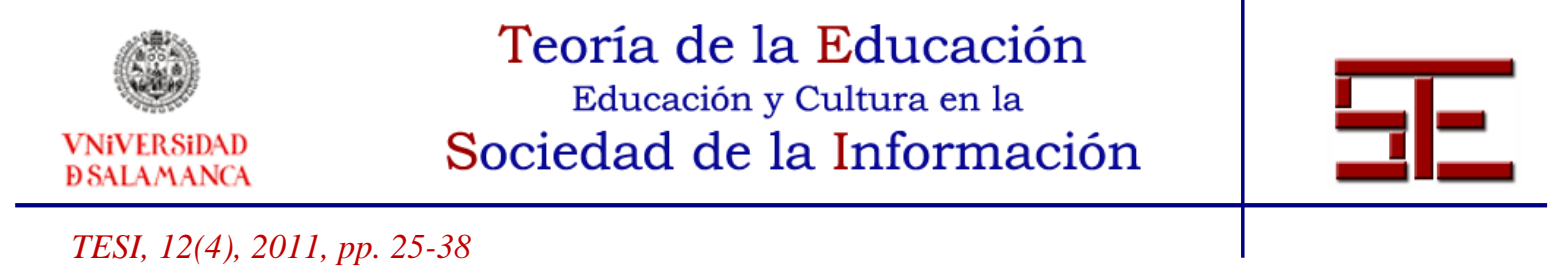

El entorno personal de aprendizaje (PLE) tiene que ser trabajado con las herramientas 2.0 por el profesorado, para favorecer la integración, gestión y control de instrumentos y para el desarrollo de nuevos métodos de aprendizaje colaborativo y cooperativo entre quienes enseñan y los sujetos que aprenden.

\section{BIBLIOGRAFÍA.}

Aajzen, I. \& Fishbein, M. (1977). Attitude-behavior relationsa theoretical análisis and review of empirical research. Psychologial Bulletin, 84 (5), 888-918.

Alba, C. (Dir.) (2004). Viabilidad de las propuestas metodológicas derivadas de la aplicación del crédito europeo por parte del profesorado de las universidades españolas, vinculadas a la utilización de las TIC en la docencia y la investigación, Madrid, Dirección General de Universidades, MEC. Extraído el 20 mayo, de 2011 de http://www.psico.uniovi.es/Fac_Psicologia/paginas_EEEs/Adaptacion_de_profesorado/tics/3-

InformeGlobal.pdf.

Anderson, P. (2007). What is Web 2.0? Ideas, technologies and implications for education, JISC, Technology and Standards Watch. Extraído el 20 de mayo, de 2011 http://www.jisc.ac.uk/media/documents/techwatch/tsw0701b.pdf.

Baelo, R. \& Arias, A. R. (2011). Formación del profesorado universitario en tecnologías de la información y la comunicación. En Maquilón, J.J., García, M.P. \& Belmonte, M.L. (Coords.), Innovación educativa en la enseñanza formal. Murcia: Edit.um.

Barros, S. (Dir.) (2004). Las TIC en el sistema universitario español. Madrid: CRUE.

Consejo de Coordinación Universitaria (2006). Propuestas para la Renovación de las Metodologías Educativas en la Universidad. Madrid: Secretaría General Técnica del $\begin{array}{llll}\text { MEC. } & \text { Extraído } & 2011\end{array}$ http://www.upcomillas.es/eees/Documentos/PROPUESTA_RENOVACION.pdf

De Pablos, J. (2008). Nuevas formas de trabajo en las aulas universitarias con el soporte de las Tecnologías de la Información. En Del Moral, M.E., \& Rodríguez, R. (Coords.), Experiencias docentes y TIC (pp. 43-58). Oviedo: Octaedro.

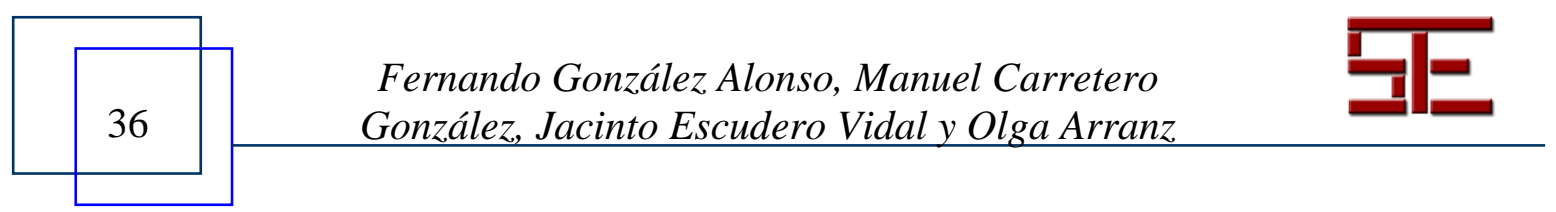




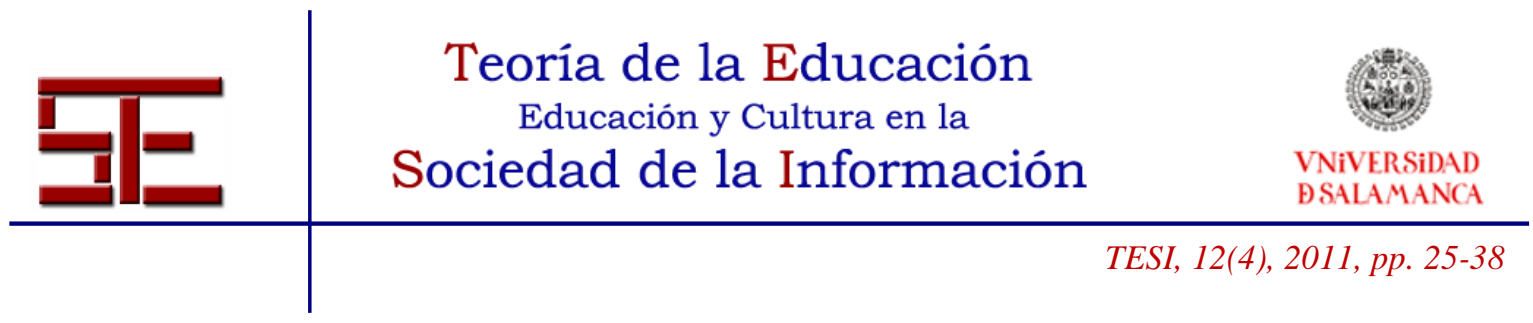

Del Moral, M. E. \& Rodríguez, R. (Coords.) (2008). Experiencias docentes y TIC. Oviedo: Octaedro.

Fernández Sánchez, M. R. \& Sosa Díaz, M.J. (2011). La docencia universitaria y las aplicaciones de la web 2.0: experiencias para innovar en el espacio europeo de educación superior. En Maquilón, J.J., García, M.P. \& Belmonte, M.L. (Coords.), Innovación educativa en la enseñanza formal (p. 788). Murcia: Edit.um.

Fernández, S. (2010). Vivir sin miedos. Atrévete a comenzar de nuevo. Barcelona: Plataforma Editorial.

Gisbert Cervera, M. (2004). La formación del profesorado para la sociedad del conocimiento. Bordón, 56 (3 y 4), 573-585.

Michavila, F. (Coord.) (2004). Las innovaciones educativas basadas en las tecnologías de la información en la formación universitaria presencial y a distancia. Dirección General de Universidades, Madrid: MEC.

Sánchez García, A. B., Pinto, J. \& González, M.A. (2011). Experiencia de formación del profesorado no universitario para la integración de la pizarra digital en el aula: estudio de los componentes cognitivo, afectivo y conductual de sus actitudes ante dicha integración. En Maquilón, J.J., García, M.P. y Belmonte, M.L. (Coords.), Innovación educativa en la enseñanza formal (pp. 445-454). Murcia: Edit.um.

\section{NOTAS:}

${ }^{1}$ Sergio Fernández López, periodista y escritor especializado en crecimiento personal y liderazgo.

2 Javier Nó Sánchez, catedrático de Tecnología de la Información y Decano en la Facultad de Comunicación de la UPSA.

${ }^{3}$ Juan Carlos Gacitúa, profesor de la Facultad de Comunicación de la UPSA.

${ }^{4}$ Roberto González Fontenla, formador, consultor y marketing 2.0.

5 Carmen González Franco, profesora de secundaria y premiada por sus blogs educativos

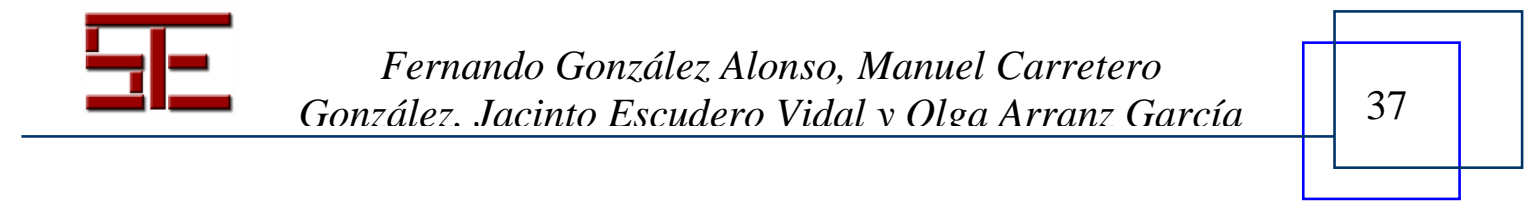




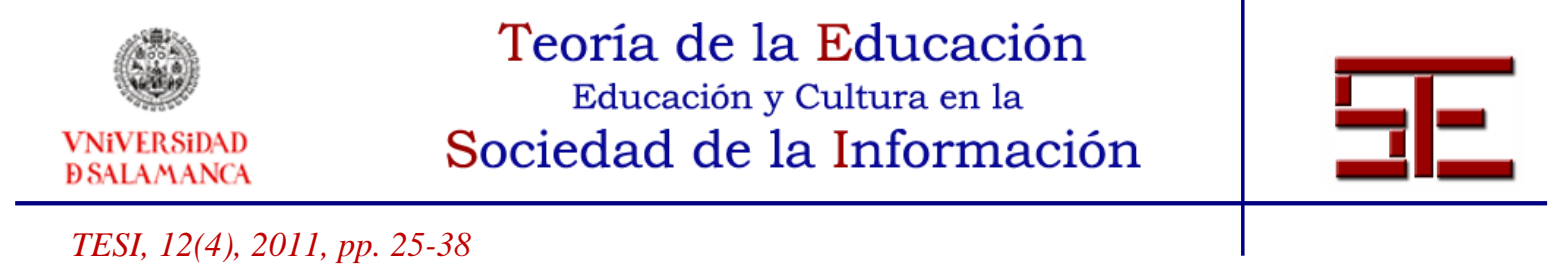

Para citar el presente artículo puede utilizar la siguiente referencia:

González Alonso, F., Carretero González, M., Escudero Vidal, J. y Arranz García, O. (2011). Niños 2.0, una experiencia formativa en actitudes y valores para el profesorado ante la web 2.0 y TIC. Revista Teoría de la Educación: Educación y Cultura en la Sociedad de la Información. 12(4), 25-37 [Fecha de consulta: dd/mm/aaaa].

http://campus.usal.es/ revistas_trabajo/index.php/revistatesi/article/view/8524/8610

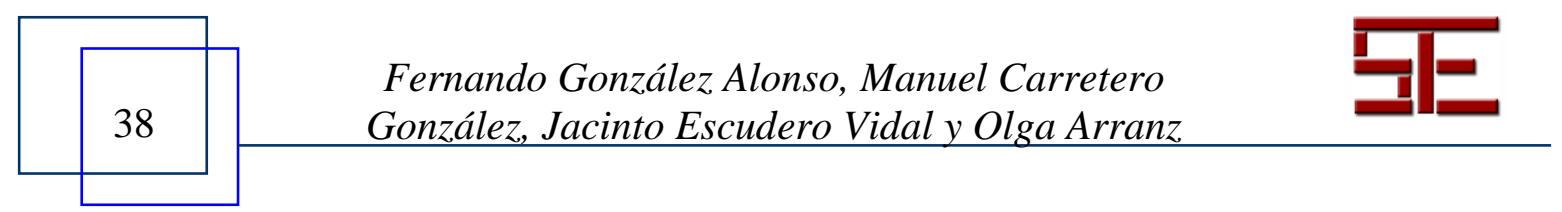

